Gut, 1982, 23, 683-691

\title{
Distribution of a liquid meal within the stomach and gastric emptying after vagotomy and drainage operations
}

\author{
O LAWAETZ, * Y ARITAS, N J G BROWN, D N L RALPHS, and E SJøNTOFT* \\ From the Department of Surgical Studies, The Middlesex Hospital and Medical School, London
}

SUMMARY The intragastric distribution of a radionuclide labelled liquid meal was studied in 62 patients with duodenal ulceration by means of a gamma camera imaging system. A total of 85 gastric emptying studies (23 preoperative and 62 postvagotomy tests) revealed three distinct patterns of distribution. The activity distribution in the stomach after vagotomy showed a slower rate of return and a decreased accumulation of the meal in the proximal part of the stomach compared with the pattern in the intact stomach. The return of the liquid meal to the proximal stomach was considered to be a physiological process controlled by vagal reflexes and changed by a vagotomy. These observations made during emptying of a liquid meal seem to give rise to a better differentiation of the motor patterns after various types of gastric operations. With a quantification of the intragastric patterns of distribution it may be possible in a prospective study to give a more graded evaluation of changes induced by vagotomy than is possible with the traditional characterisation of gastric emptying.

In 1898 Cannon $^{1}$ made the interesting observation that the cardiac portion of the stomach acts as an active reservoir. This was achieved by use of $X$-ray examination and a nutritional semi-solid contrast medium. Cannon and $\mathrm{Lieb}^{2}$ described this reservoir function in non-vagotomised cats as an ability to accommodate an ingested meal in the proximal stomach with only a slight rise in the intragastric pressure. The reservoir function was evident in response to both solid and liquid meals. ${ }^{3}$ In dogs, Wilbur and $\mathrm{Kelly}^{4}$ found that the intragastric pressure rose with distension in the vagotomised stomach. In man, intragastric pressure measurements have revealed similar gastric responses to expansion. ${ }^{5-7}$ The distribution of the gastric contents within the stomach after vagotomy, however, has not been described.

In recent years radionuclide labelled meals have been used on an increasing scale for assessment of gastric emptying. Since Harvey et $^{8} \mathrm{l}^{8}$ introduced the use of the gamma camera for this type of examination, improved storage-display-imaging systems

\footnotetext{
* Present address: Institute for Experimental Research in Surgery, University of Copenhagen, 71, Nørre Alle, DK-2100 Copenhagen $\emptyset$.

Received for publication 30 November 1981
}

have enabled a continuous observation of the movements of the isotope within the stomach.

In the course of measuring the gastric emptying of a labelled hypertonic glucose solution by means of a gamma camera it became apparent that, in a number of patients, the distribution of the labelled meal in the stomach after vagotomy differed from that observed preoperatively in patients with duodenal ulceration. This report describes the patterns of distribution of labelled fluid meals in the stomach before and after vagotomy with or without a drainage operation. To allow comparisons of these phenomena the intragastric patterns of distribution are quantified and expressed by a distribution index (DI).

\section{Methods}

\section{PATIENTS}

A total of 85 gastric emptying tests (23 preoperative, 13 early postoperative, and 49 late postoperative tests) were performed on 62 patients ( 38 men and 24 women with a mean age of 45 years) with a history of duodenal ulceration. Twenty-three patients were studied twice, eight patients before and after operation, and another 15 on two postoperative occasions. In addition to the late postoperative 
gastric emptying tests, an early test was performed within a few weeks of operation on 13 patients.

The interval between operation and the late postoperative test ranged from three months to 108 months with a mean of 23 months. Twenty-two patients had had a vagotomy (truncal, 17; selective gastric, two; proximal gastric, three) and a drainage operation (Heineke-Miculicz pyloroplasty, 18; gastroenterostomy, four). Twenty-seven patients had had a vagotomy alone (selective gastric, 11; proximal gastric, 16).

To assess the day-to-day reproducibility of the observations gastric emptying tests were repeated at one-day intervals on four patients at least 10 days after laparotomy for cholecystectomy.

\section{Procedure}

After an overnight fast gastric emptying of a meal of $150 \mathrm{ml} 50 \%$ glucose solution labelled with $1.5 \mathrm{mCi}$ ${ }^{113}$ In chelated with DTPA (diethylene triamine pentaacetic acid) ${ }^{9}$ was studied with the patient lying in a semirecumbent position in front of a gamma camera. A parallel hole medium energy (maximum, $412 \mathrm{KeV}$ ) collimator was used. A computer connected to the gamma camera accumulated the scintigraphic data from the entire camera field of view in 60 second frames (sequential images) for 60 minutes from the start of the ingestion of the meal. At the end of the test each frame image was displayed on a television screen. The stomach, the proximal stomach, and the antrum were manually outlined on the screen using one of the early frame images (Fig. 1).

Thus four regions of interest were created corresponding to the entire field of view and the three stomach regions. Four time-activity curves were generated and corrected for physical decay of the isotope. The entire field maximum one-minute count was taken as representing the total volume of the meal ingested by the patient, and the oneminute counts obtained from the three stomach regions were expressed as percentages of this volume at given times.

Using the one-minute counts obtained from the total stomach region and the proximal stomach region, a computer programme calculated a distribution index for each gastric emptying test. This index is the intercept of a linear regression from the one-minute ratios (Fig. 2): the proportional reduction of the meal in the proximal stomach to the proportional reduction of the meal in the total stomach for 20 to 50 minutes after ingestion. In more physiological terms the distribution index, as defined in Fig. 2, varies not only with the time during which the meal is retained in the proximal

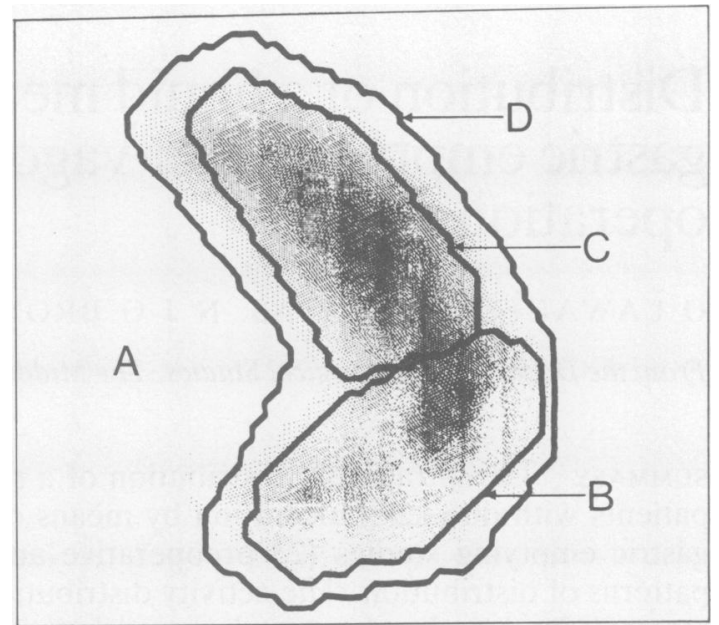

Fig. 1 Regions of interest for curve generation. Entire field of view $(A)$, antrum $(B)$, proximal stomach $(C)$, and stomach $(D)$.

stomach, but also with the rate of gastric emptying.

Gastric emptying was characterised quantitatively by the percentage obtained from the total stomach region. Because of imperfections in collimation some septal penetration of radiation occurred producing errors, which resulted in an overestimation of these percentages. A correction was performed on the basis of phantom studies (to be

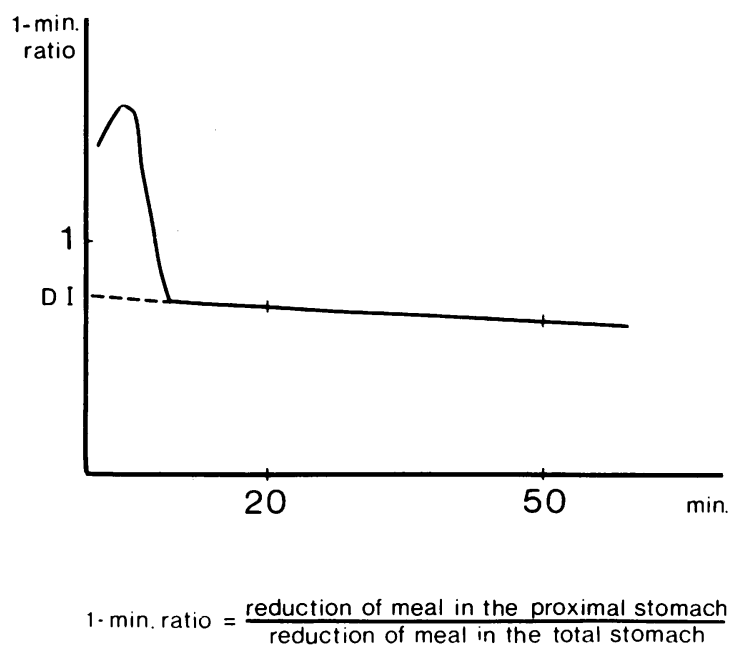

Fig. 2 Definition of distribution index (DI). One-minute ratios plotted against time. Broken line: linear regression for 20-50 minutes after ingestion. 
published). The mean percentage gastric emptying at 10-minute intervals was calculated for the preoperative patients, the patients with vagotomy and a drainage operation $(\mathrm{V}+\mathrm{D})$, and those with a vagotomy alone $(\mathrm{V})$.

The statistical method used for comparing gastric emptying data was Student's $t$ test for unpaired data. Significant differences were inferred when $p$ values were less than 0.05 .

\section{Results}

\section{PATTERNS OF DISTRIBUTION}

The activity distribution within the stomach was observed from the sequential one-minute images of the stomach, and three distinct types could be identified in the patterns of distribution of the gastric contents.

\section{Type 1 pattern}

This pattern is shown in Fig. 3a. Immediately after ingestion most of the meal is in the dependent part of the stomach. Within the next few minutes a major part of the meal moves back to the proximal stomach and largely remains there until it leaves the stomach at a constant rate at between 10 and 20 minutes after ingestion. Fig. 3b shows the corresponding time-activity curves from the total camera field of view (A), the antrum (B), the proximal stomach (C), and the stomach (D). The curves B and $C$ reflect the early return of the gastric contents from the antrum to the proximal stomach and the subsequent emptying into the duodenum.

This type 1 pattern predominated in the preoperative group, but was also found postoperatively. The rate at which the meal returns into the proximal stomach, as described in this pattern,
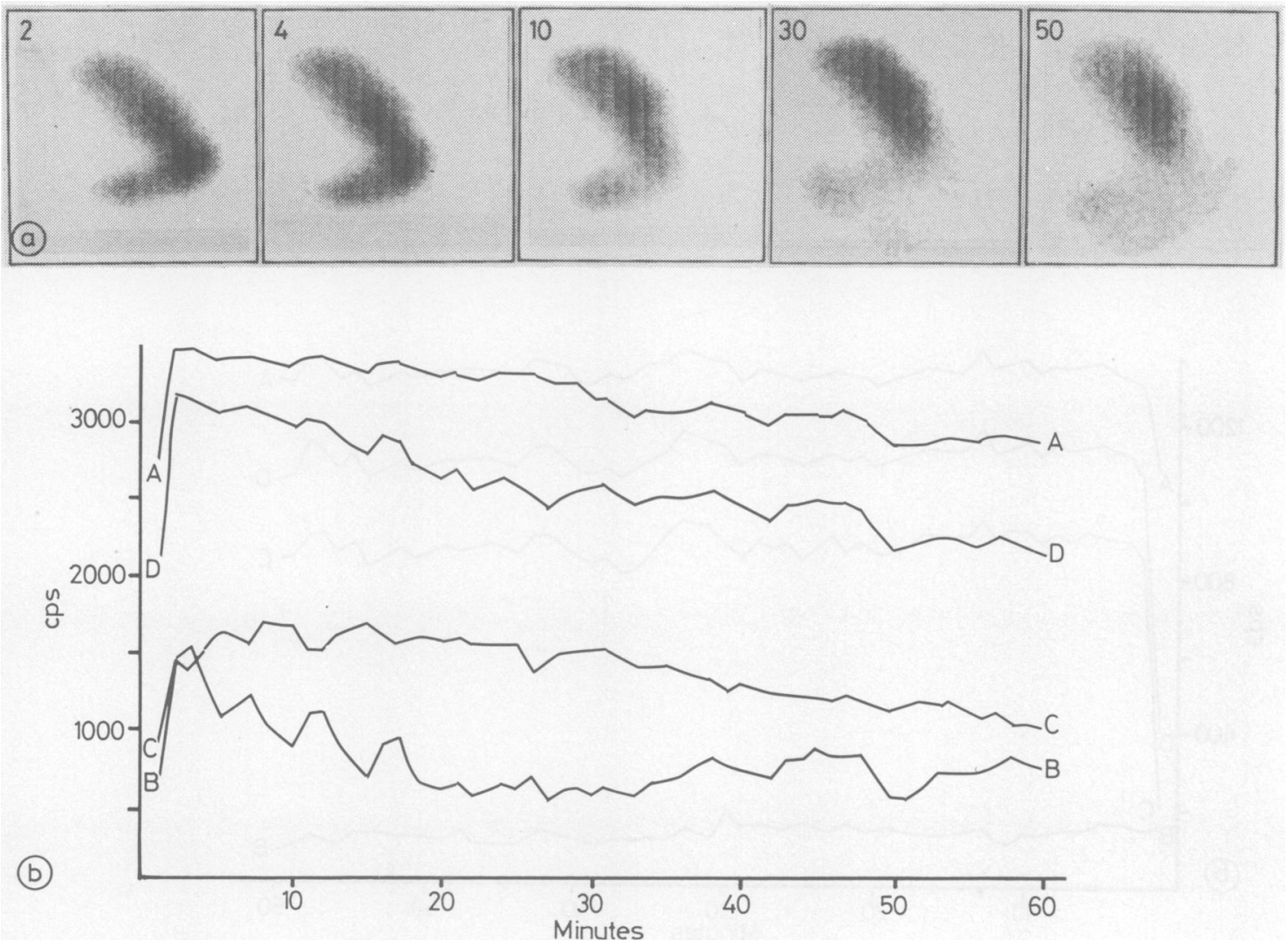

Fig. 3 (a) Type 1 pattern of activity distribution: Serial one-minute frame images at two, four, 10, 30, and 50 minutes after intake of meal from preoperative patient with duodenal ulceration. (b) Corresponding time-activity curves generated from regions of interest as shown in Fig. 1. 
varies from one subject to another. In general the return to the proximal stomach has been found to be faster and more complete in the preoperative group than in the postvagotomy groups late after operation.

\section{Type 2 pattern}

This pattern is shown in Fig. 4a. Already in the second minute after ingestion, a substantial amount of the meal is found in the proximal stomach, whence it leaves at a constant rate similar to that described in the type 1 pattern. The time-activity curves in Fig. $4 \mathrm{~b}$ demonstrate the immediate accumulation of the meal in the proximal stomach.

This pattern was observed in a number of patients in the early postoperative period. In the late postoperative period the type 2 pattern was observed in only one patient.

\section{Type 3 pattern}

This pattern is shown in Fig. 5a. Immediately after ingestion a major part of the meal appears in the bottom of the stomach, where it remains until leaving the stomach. The time-activity curves in Fig. $5 b$ show a rapid early evacuation from the various parts of the stomach followed by a steady emptying.

The type 3 pattern was observed in the postvagotomy groups and predominantly in patients with a rapid initial emptying. A similar location of the gastric contents in the bottom of the stomach was also observed in some of the early postoperative tests. The pattern was then, however, attended by a negligible emptying in contrast with the rapid early emptying in the late postoperative tests.

Eight patients were studied before and after vagotomy without a drainage operation (proximal gastric vagotomy, four; selective gastric vagotomy,
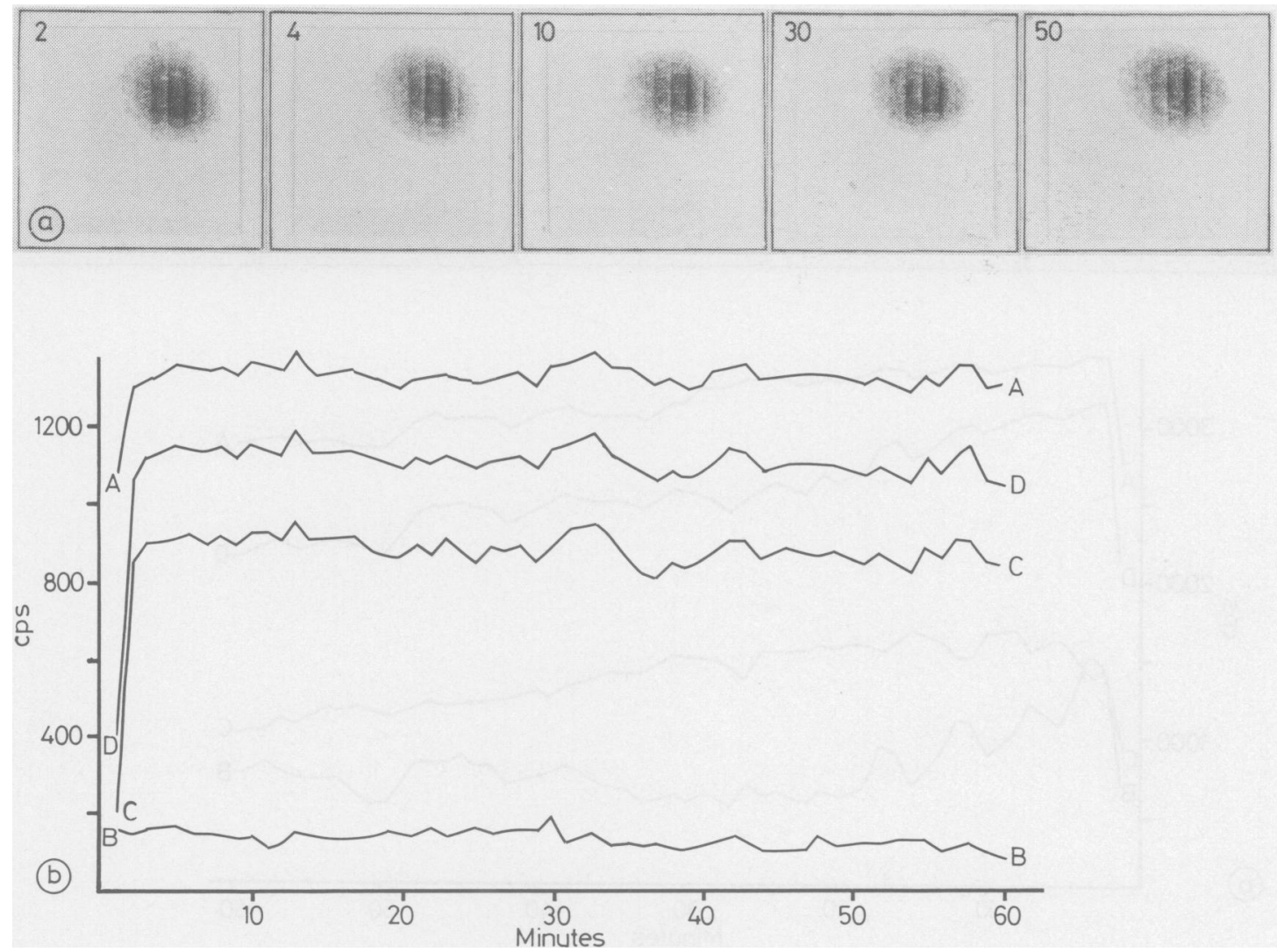

Fig. 4 (a) Type 2 pattern of activity distribution: Serial one-minute frame images at two, four, 10, 30, and 50 minutes after intake of meal from patient with early postoperative test after proximal gastric vagotomy. Antrum not depicted on any frame images indicating very small proportion of activity in this area. Antrum was clearly identified in preoperative test. (b) Corresponding time-activity curves generated from regions of interest as shown in Fig. 1. 

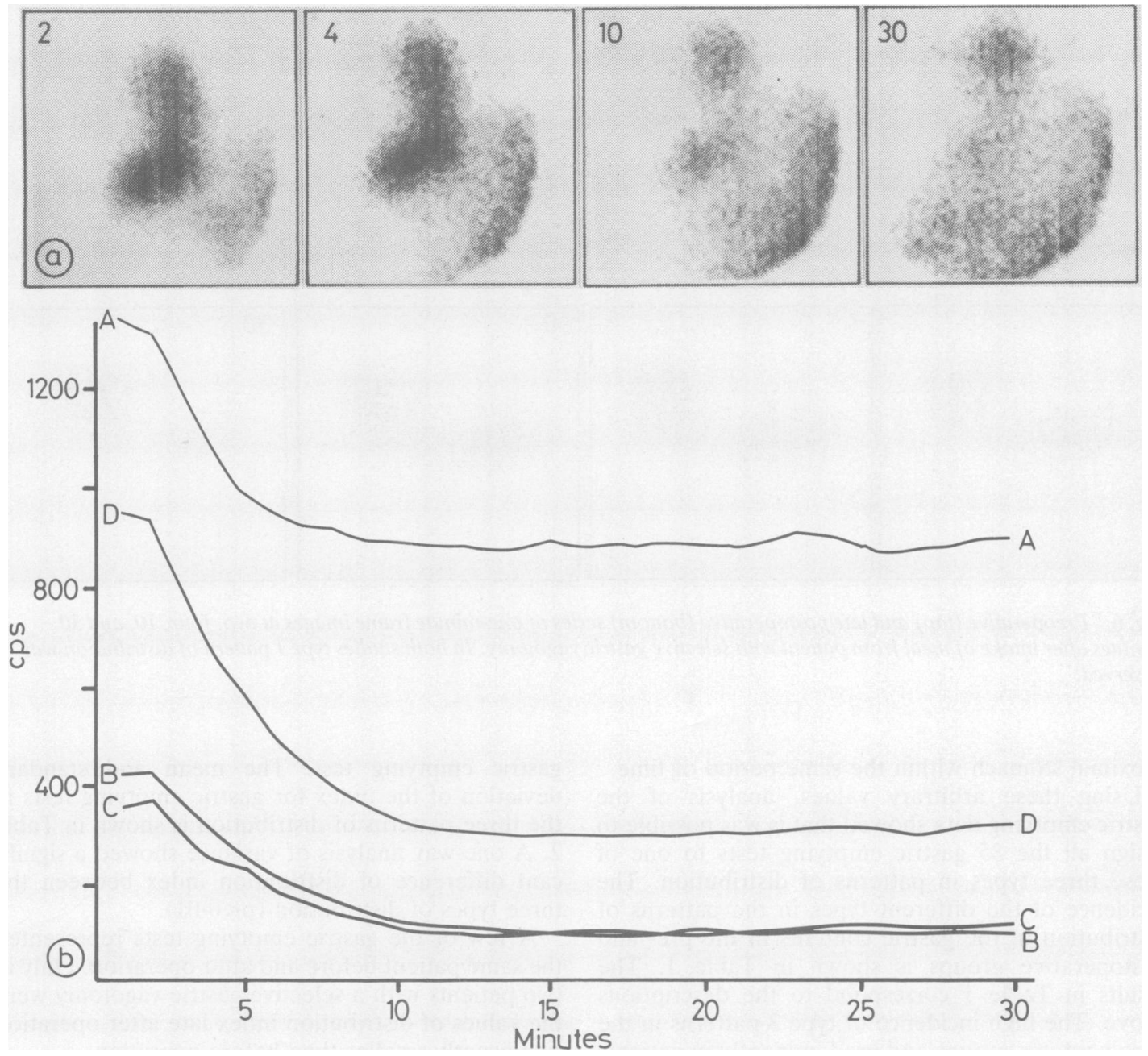

Fig. 5 (a) Type 3 pattern of activity distribution: serial one-minute frame images at two, four, 10, and 30 minutes after intake of meal from patient three months after selective gastric vagotomy. (b) Corresponding time-activity curves generated from regions of interest as shown in Fig. 1.

four). Comparisons between the individual images and time-activity curves from the preoperative and the late postoperative studies confirmed in these patients that the return of the gastric contents to the proximal stomach was delayed, and accumulation impaired in the vagotomised stomach. Preoperative and late postoperative images from a patient with a selective gastric vagotomy are shown in Fig. 6.

The 13 early postoperative tests were characterised by slow gastric emptying and all three types of distribution. The images from an early postoperative test are shown in Fig. 7. They indicate a type 2 pattern of distribution with an immediate accumulation in the proximal stomach followed by a slow evacuation to the antrum and further into the duodenum.

\section{DISTRIBUTION INDEX}

Gastric emptying tests in which one-third to twothirds of the total meal was retained in the proximal stomach by 20 minutes after ingestion were considered to belong to type 1 pattern, whereas type 2 and type 3 patterns implied that a greater and a smaller fraction, respectively, were retained in the 

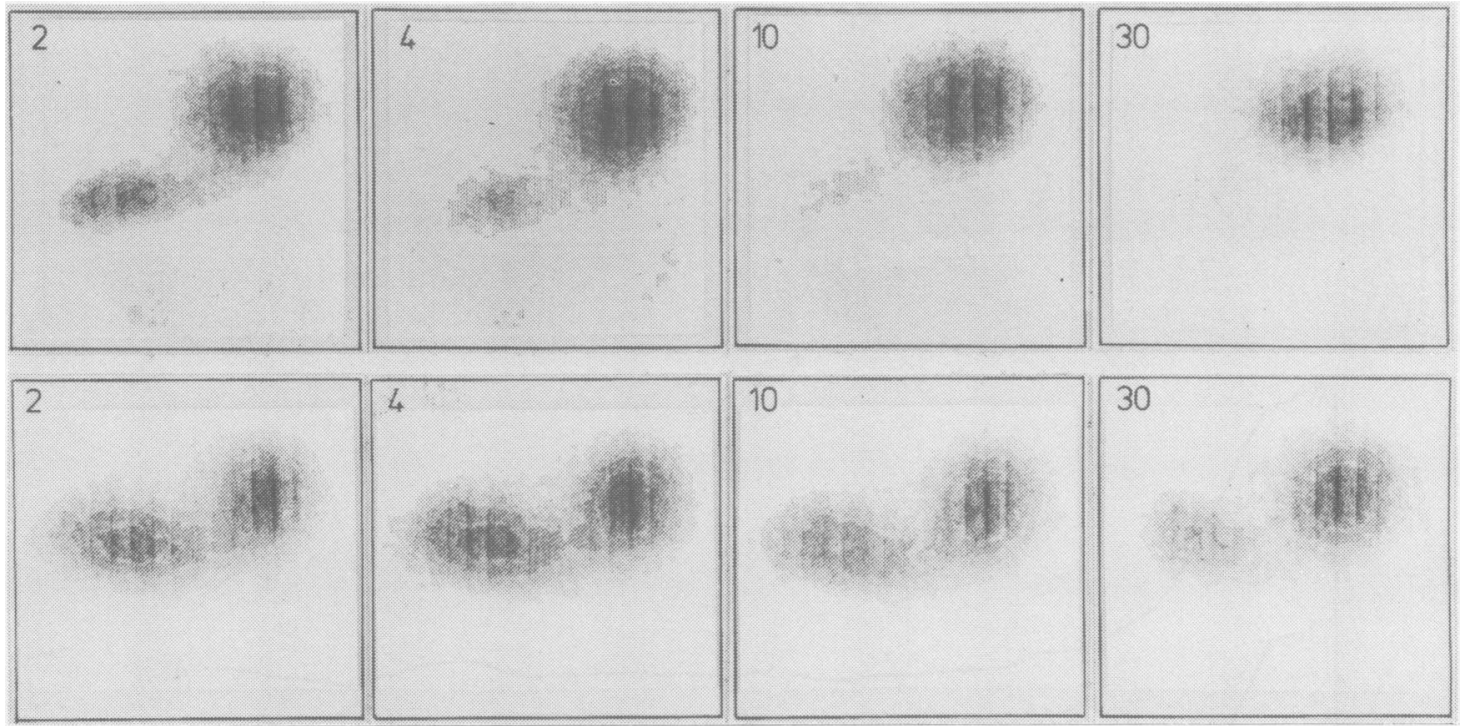

Fig. 6 Preoperative (top) and late postoperative (bottom) series of one-minute frame images at two, four, 10, and 30 minutes after intake of meal from patient with selective gastric vagotomy. In both studies type 1 pattern of distribution was observed.

proximal stomach within the same period of time.

Using these arbitrary values, analysis of the gastric emptying data showed that is was possible to assign all the 85 gastric emptying tests to one of these three types in patterns of distribution. The incidence of the different types in the patterns of distribution of the gastric contents in the pre- and postoperative groups is shown in Table 1 . The results in Table 1 correspond to the descriptions above. The high incidence of type 3 patterns in the postvagotomy groups and predominantly in patients who had the whole stomach vagally denervated in contrast with the high incidence of type 1 patterns in the preoperative group is highly significant $\left(\chi^{2}=39 \cdot 25, \mathrm{df}=6, \mathrm{p}<10^{-5}\right)$.

The distribution index was calculated for each gastric emptying test. The mean and standard deviation of the index for gastric emptying tests in the three patterns of distribution is shown in Table 2. A one-way analysis of variance showed a significant difference of distribution index between the three types of distribution $(\mathrm{p}<0 \cdot 01)$.

A few of the gastric emptying tests represented the same patient before and after operation. Only in two patients with a selective gastric vagotomy were the values of distribution index late after operation significantly smaller than before operation.

\section{DAY-TO-DAY REPRODUCIBILITY}

Both type 1 and the type 2 patterns of distribution of the gastric contents were observed. Neither on the frame images nor on the time-activity curves was it

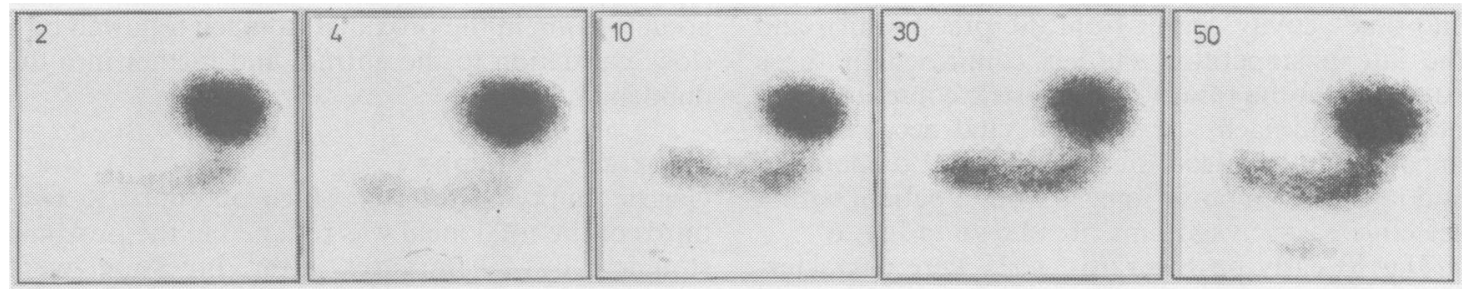

Fig. 7 Early postoperative one-minute frame images at two, four, 10,30, and 50 minutes after intake of meal from patient in Fig. 5 . 
Table 1 Incidence of types in patterns of distribution of gastric contents in four groups of patients

\begin{tabular}{|c|c|c|c|c|}
\hline & \multirow{2}{*}{$\begin{array}{l}\text { Tests } \\
\text { (no.) }\end{array}$} & \multicolumn{3}{|c|}{ Type of distribution } \\
\hline & & 1 & 2 & 3 \\
\hline Preoperative & 23 & 20 & 0 & 3 \\
\hline Early postoperative & 13 & 5 & 5 & 3 \\
\hline \multicolumn{5}{|l|}{ Late postoperative } \\
\hline Vagotomy & 27 & 17 & 1 & 9 \\
\hline Vagotomy + drainage & 22 & 7 & 0 & 15 \\
\hline
\end{tabular}

possible to demonstrate any differences between the gastric emptying tests, repeated at one-day intervals in the four patients after cholecystectomy.

The estimates of distribution index of the duplicate gastric emptying tests are shown in Table 3. No systematic differences between the first and second estimates were found.

\section{GASTRIC EMPTYING}

The results of gastric emptying are shown in Fig. 8. In the vagotomy + drainage group the mean percentage emptied was significantly greater than in the preoperative group and in the vagotomy group. The faster emptying in the vagotomy + drainage group was due to the rate of emptying in the initial 10-minute period of observation. The mean percentage emptied between 10 minutes and 60 minutes did not differ significantly from that in the two other groups. In the vagatomy group the emptying at no time differed significantly from the mean of the preoperative values.

\section{Discussion}

The measurements of gastric emptying revealed a considerable variation in the shape and the size of the stomach from one subject to another. The frame images allowed observation of changes in distribution of gastric activity. The time-activity curves from the antrum and the proximal stomach are true reflections of movements within the stomach rather than produced by changes in the counting efficiency due to geometrical variations. This is indicated by the count stability in the entire field of view (curve A

Table 2 Mean and standard deviation of distribution index for three types in patterns of distribution

\begin{tabular}{llll}
\hline & \multicolumn{4}{l}{ Type of distribution } \\
\cline { 2 - 4 } & 1 & 2 & 3 \\
\hline Mean distribution index & 0.57 & 1.00 & 0.33 \\
Standard deviation & 0.10 & 0.14 & 0.05 \\
Gastric emptying tests (no.) & 49 & 6 & 30 \\
\hline
\end{tabular}

Table 3 Distribution index of duplicate gastric emptying tests

\begin{tabular}{lll}
\hline & \multicolumn{2}{l}{ Distribution Index } \\
\cline { 2 - 3 } Subject & Test 1 & Test 2 \\
\hline A & 0.61 & 0.59 \\
B & 0.58 & 0.60 \\
C & $1 \cdot 14$ & 1.14 \\
D & 0.78 & 0.74 \\
\hline
\end{tabular}

in Fig. 3b) during the early dislocation of activity from the antrum to the proximal stomach.

In the majority of the preoperative and postoperative studies the partition of activity in the proximal and distal stomach was distinct. The two regions were separated by a line drawn through the lesser and the greater curvature some one-third to one-half of the distance from the oesophagus to the pylorus. The variation in the creation of the regions of interest has been studied by repeating the procedure on the same frame image at intervals of a few days, weeks, and months. The minor variations in the shape and size of the regions of interest that were observed did not give rise to changes in the character of the time-activity curves.

The division described above corresponds to Kelly's physiological division of the stomach into two functional regions: ${ }^{10}$ the proximal stomach, which acts as an active reservoir, and the antrum, which acts as a mixer and grinder of the gastric

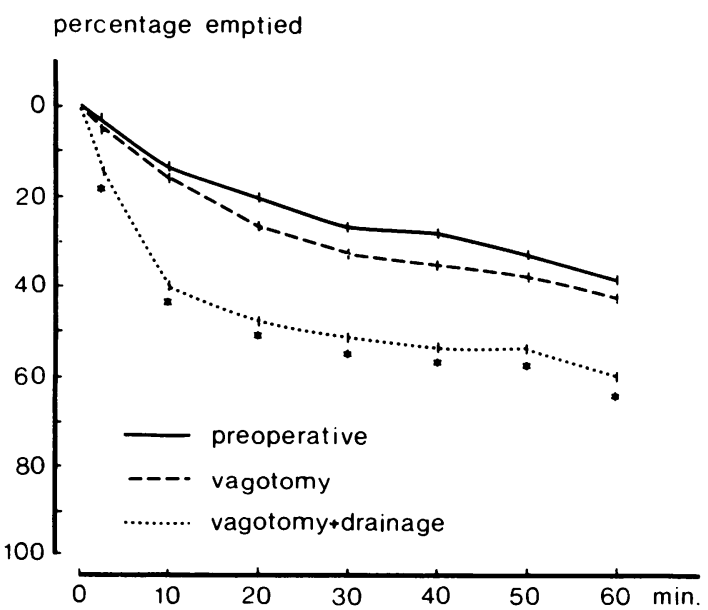

Fig. 8 Percentage of liquid meal emptied against time. Points are means $\pm S E M$. Curve of vagotomy + drainage group is significantly different from both preoperative and vagotomy alone groups. ${ }^{*} p<0.001$ (unpaired t test). 
contents. Gastric motility is characterised by sustained proximal contractions, peristaltic waves, and terminal antral contractions. The proximal gastric contractions are considered to regulate the intragastric pressure and are thus involved in the gastric emptying of liquids. This function is mediated by vagal reflexes and abolished by vagotomy. In the regulation of liquid gastric emptying the peristaltic waves and the terminal antral contractions are considered to be of only minor importance. ${ }^{10}$

The return of gastric contents to the proximal stomach (retropulsion) is a well-known phenomenon in the trituration process of solids. ${ }^{110}$ This process is impaired by vagal denervation of the antrum. ${ }^{10}$ Retropulsion of fluid meals has also been observed by Barker et al. ${ }^{11}$ Studying gastric emptying in patients with systemic sclerosis Barker found evidence of mass retropulsion of a radionuclide labelled fluid meal from the antrum into the proximal stomach in two patients. The phenomenon was considered as a pathological process rather than a physiological one. ${ }^{11}$

In the present series of patients with duodenal ulceration the activity distribution in the preoperative stomach exhibited an early accumulation of the activity in the proximal stomach (type 1 and type 2 patterns of distribution).

The early postoperative stomach was characterised by a persistant location of the meal in the proximal stomach (type 1 and 2 patterns) or in the antrum (type 3 pattern) and a slow gastric emptying. These observations indicated less motor activity in the early postoperative stomach than in the late postoperative one. This is consistent with our practical experience in the clinic.

Late after operation the postvagotomy stomach was characterised by a persistence of activity in the antrum (type 3 pattern of distribution) followed eventually by a delayed return to the proximal stomach (type 1 pattern of distribution). The patterns were attended by rapid early emptying. No attempt has been made to evaluate the three types of distribution in relation to the occurrence of postvagotomy symptoms.

The proximal vagal fibres were severed in all three types of vagotomy. Therefore the slower rate of return and the decreased accumulation in the proximal stomach of liquid gastric contents may be explained solely by the disruption of the proximal vagal reflexes modulating the gastric reservoir function. The type 3 pattern of distribution with rapid gastric emptying was found predominantly in the vagotomy + drainage group. When it is remembered that 19 out of 22 patients in this group also had the antrum vagally denervated, this seems to point to the antrum as having a greater influence on the control of liquid meals than indicated by Kelly. ${ }^{10}$ Because of the different vagotomies the data do not allow of any hypothesis to be formulated regarding the influence of the drainage operation upon the distribution of the fluid meal.

The return of the liquid meal and the accumulation into the proximal stomach, as observed in this study, is considered to be a physiological process controlled by vagal reflexes and changed by a vagotomy. An unambiguous relationship between the integrity of the vagal nerves and the activity distribution within the stomach has not been proved in the paper.

This study has demonstrated that the intragastric distribution of a labelled fluid meal in the stomach after vagotomy differs from that in the intact stomach. These observations made during emptying of a liquid meal give rise to a better differentiation of the motor patterns after various types of gastric operations. When the intragastric patterns of distribution observed (movements and location of the meal within the stomach) are quantified it may be possible in a prospective study to give a more graded evaluation of changes in the motor patterns induced by proximal gastric, selective gastric, or truncal vagotomies than is possible with the traditional characterisation of gastric emptying (emptying time or emptying rate).

\section{References}

1 Cannon W B. The movements of the stomach studied by means of the röntgen rays. Am J Physiol 1898; 1: 359-82.

2 Cannon $\mathrm{W} \mathrm{B}$, Lieb $\mathrm{C} \mathrm{W}$. The receptive relaxation of the stomach. Am J Physiol 1911; 29: 267-73.

3 Donovan I A. Gastric emptying. Thesis: University of Birmingham, 1976: 23.

4 Wilbur B G, Kelly K A. Effect of proximal gastric, complete gastric, and truncal vagotomy on canine gastric electric activity, motility, and emptying. Ann Surg 1973; 178: 295-302.

5 Jahnberg T, Martinson J, Hultén J, Faster S. Dynamic gastric response to expansion before and after vagotomy. Scand J Gastroenterol 1975; 10: 593-98.

6 Köster W, Madsen P. The intragastric pressure before and immediately after truncal vagotomy. Scand $J$ Gastroenterol 1970; 5: 381-83.

7 Stadaas J, Aune S. Intragastric pressure/volume relationship before and after vagotomy. Acta Chir 
Scand 1970; 136: 611-15.

8 Harvey R, Mackie D B, Brown N J et al. Measurement of gastric emptying time with a gamma camera. Lancet 1970; 1: 16-18.

9 Ralphs D N L, Thompson J P S, Haynes S et al. The relationship between the rate of gastric emptying and the dumping syndrome. Br J Surg 1978; 65: 637-41.

10 Kelly K A. Gastric motility after gastric operations. Surg Ann 1974; 6: 103-23.

11 Barker M C J, Cobden I, Axon A T R. Proximal stomach and antrum in stomach emptying. Gut 1979; 20: $309-11$. 